\title{
Site or Size of Waist Circumference, Which one is More important in Metabolic Syndrome?
}

\author{
Syed Mohd Razi Gutch Manish, Gupta Kumar Keshav, Kumar Sukriti, Abhinav Gupta
}

\section{Syed Mohd Razi Gutch Manish', Gupta Kumar Keshav', Kumar Sukriti', Abhinav Gupta'}

\section{Dept of endocrinology, LLRM Medical College, Meerut, UP, INDIA. \\ ${ }^{2}$ Department of Radiodiagnosis, SGPGI, Lucknow, INDIA.}

\section{Correspondence}

Manish Gutch, D-15, LLRM Medical College, Meerut, Uttar Pradesh, INDIA.

Phone no: 945-342-9252

Email: manish07gutch@gmail.com

\section{History}

- Submission Date: 31-12-15;

- Review completed: 03-05-16;

- Accepted Date: 06-05-16.

\section{DOI : 10.5530/ijmedph.2016.2.4}

\section{Article Available online}

http://www.ijmedph.org/v6/i2

\section{Copyright}

(C) 2016 Phcog.Net. This is an open-access article distributed under the terms of the Creative Commons Attribution 4.0 International license.

\begin{abstract}
Abdominal obesity is one of the pivotal factors in defining the metabolic syndrome. Abdominal obesity is assessed by the various clinical surrogates among which waist circumference is considered to be simple, inexpensive \& sensitive tool. But various controversies surround the exact cut offs and the ideal sites of waist circumference measurement, so in this review we discussed these issues.

Key words: Metabolic syndrome, Waist circumference, Cardio metabolic risk, Abdominal obesity, Harmonized Definition.
\end{abstract}

\section{INTRODUCTION}

Metabolic syndrome is a complex disorder and is characterized by clustering of a number of interrelated factors increasing the risk of coronary heart disease (CHD) and Type 2 Diabetes mellitus (T2DM). The defining components of metabolic syndrome include dyslipidemia (elevated triglycerides and apolipoprotein B (apoB)-containing lipoproteins, and low high-density lipoproteins (HDL)), elevation of arterial blood pressure (BP), dysregulated glucose homeostasis, abdominal obesity and/or insulin resistance (IR). ${ }^{1}$ Abdominal obesity is one of the pivotal features in the pathogenesis of metabolic syndrome. In order to clearly define metabolic syndrome, it is the matter of prime importance to device simple clinical measures for precise measurement of abdominal obesity.

Since the first definition of metabolic syndrome by WHO, ${ }^{2}$ to the most recent Harmonized definition, ${ }^{3}$ various clinical tools ranging from waist -hip ratio to waist circumference have been used to quantify central obesity. Among the measures of central obesity waist circumference is considered to be simple \& inexpensive measure with excellent correlation with abdominal imaging and is used in all definitions of metabolic syndrome except WHO criteria, as surrogate marker of central obesity. ${ }^{4}$

Waist circumference appears to better central obesity indicator than BMI and waist hip ratio. ${ }^{5}$ Despite waist circumference being one of the basic components of every definition of metabolic syndrome, the ideal site \& size which can define all the $\mathrm{CV}$ risk is still a matter of debate.

\section{FINDING THE MAGICAL NUMBERS}

The ideal waist measurement above which the risk of CVD \& T2 DM increases significantly is not well defined since its introduction in the various definitions of the metabolic syndrome. The diagnostic cut offs of waist circumference used in the various guidelines are the result of expert deliberations but not the evidence based process \& epidemiological studies. " "Same doesn't fit all", so the same cut off for metabolic syndrome can't be applied to all the ethnicities of the world having different genetic makeup, body fat content \& distribution, environmental factors and life style which affect their susceptibility for metabolic syndrome. For example Asians tend to have greater body fat for the same BMI when compared with Caucasians so Asians develop hypertension, T2DM and dyslipidemia at a lower BMI. ${ }^{5}$ This fact was realized very late in 2005 when IDF, 7 proposed different cut off for the waist circumference for the first time, as all previous definitions had same cut off for all the ethnicities. Though all the definitions coming after IDF, ${ }^{7}$ definition have different cut offs for different ethnicities but the exact increase in the risk of CVD \& T2DM is not well defined. A study entitled "Comparisons of waist circumferences measured at 4 sites" done by J. Wang et al. in 2003 compared waist circumference at four different sites in 49 males and 62 females. In this study author measured the waist circumference at following sites: 1 . Immediately below the lowest rib (WC1); 2. At the narrowest waist (WC2, recommended in the Anthropometric Standardization Reference Manual); 3. Midpoint between the lowest rib and iliac crest (WC3, recommended in the World Health Organization (WHO) guidelines) and 4. Immediately above the iliac crest (WC4, Recommended in the National Institutes of Health (NIH) 
guidelines and applied in the third National Health and Nutrition Examination Survey (NHANES III)). They found that mean values of waist circumference were WC2 $<\mathrm{WC} 1<\mathrm{WC} 3<\mathrm{WC} 4(\mathrm{P}<0.01)$ in females and $\mathrm{WC} 2<\mathrm{WC} 1, \mathrm{WC} 3$, and WC4 $(\mathrm{P}<0.01)$ in males. They found that for all 4 sites measurement reproducibility was high, with intra-class correlation ( $\mathrm{r}$ ) values $>0.99$. WC values were significantly correlated with fatness; correlations with trunk fat were higher than correlations with total body fat in both sexes. ${ }^{8}$ The waist circumference cut offs used in various guidelines are shown in Table 1

\section{FINDING THE RIGHT SITE}

As there are numerous controversies regarding the ideal cut off for waist circumference similar is the case for the ideal site for waist circumference measurement. Different waist circumference measuring sites are taken for different studies \& guidelines. There are 10 documented sites noted in literature by Guerra et al. as shown in table 2, (1) Narrowest point between the iliac crest and the lower rib margin; (2) Midway between the lower rib margin and the iliac crest; (3) Narrowest point between the umbilicus and the xiphoid process; (4) One-third of the distance between the xiphoid process and the umbilicus; (5) Midway between the xiphoid process and the umbilicus; (6) Widest diameter between the xiphoid process and the iliac crest; (7) At the level of the iliac crest; (9) At the level of the umbilicus; (10) $2.5 \mathrm{~cm}$ above the umbilicus and (11) At the lower border of the 10thrib. ${ }^{12}$ But the ideal site should be sensitive enough to point out the population at CV risk. One uniform site for waist circumference measurement is needed to bring about uniformity in diagnosis criteria, prevalence studies \& intervention outcomes. There are various studies which focus on this issue that which site corresponds better to the CV risk \& metabolic syndrome. In 2010, Mason et al. compared 4 sites of waist measurement namely iliac crest, midpoint, umbilicus, and minimal waist and found that more men \& women met the criteria of metabolic syndrome when waist circumference was measured at umblicus, although correlation of CV risk \& WC didn't varied significantly between the different sites. ${ }^{13} \mathrm{~A}$ recent scientific statement issued in 2011 by AHA advocate WC measurement at iliac crest as it is the easiest \& most consistent location. ${ }^{4}$ R. S. Guerra et al. 2012, conducted a study on elderly subjects to find

Table 1: The waist circumference cut offs used in various guidelines $\{14\}$

\begin{tabular}{|c|c|c|c|}
\hline \multicolumn{2}{|c|}{ Criteria } & Male & Female \\
\hline \multicolumn{2}{|c|}{ 1. European Group on Insulin resistance (EGIR)1999 [9] } & $>94 \mathrm{~cm}$ & $>80 \mathrm{~cm}$ \\
\hline \multicolumn{2}{|c|}{$\begin{array}{l}\text { 2. National Cholesterol Education Program / Adult Treatment Panel III (NCEP/ATP } \\
\text { III 2001) }[10]\end{array}$} & $>102 \mathrm{~cm}$ & $>88 \mathrm{~cm}$ \\
\hline \multicolumn{2}{|c|}{ 3. Updated NCEP/ATP III (2004) [11] } & $>102 \mathrm{~cm}$ & $>88 \mathrm{~cm}$ \\
\hline \multicolumn{4}{|c|}{ 4. International Diabetes Federation (IDF2005)[7]: Waist circumference was the essential criteria \& was ethnicity based for the first time: } \\
\hline \multicolumn{4}{|c|}{$\begin{array}{l}\text { Waist circumference } \\
\text { (as measure of central obesity) }\end{array}$} \\
\hline \multicolumn{2}{|c|}{ Country/ethnic group } & Male & Female \\
\hline \multicolumn{2}{|c|}{ Europids } & $\geq 94 \mathrm{~cm}$ & $\geq 80 \mathrm{~cm}$ \\
\hline \multicolumn{2}{|c|}{ South Asians } & $\geq 90 \mathrm{~cm}$ & $\geq 80 \mathrm{~cm}$ \\
\hline \multicolumn{2}{|c|}{ Chinese } & $\geq 90 \mathrm{~cm}$ & $\geq 80 \mathrm{~cm}$ \\
\hline \multicolumn{2}{|c|}{ Japanese } & $\geq 85 \mathrm{~cm}$ & $\geq 90 \mathrm{~cm}$ \\
\hline \multicolumn{2}{|c|}{ Ethnic South and Central Americans } & \multicolumn{2}{|c|}{$\begin{array}{l}\text { Use South Asian recommendations until more specific } \\
\text { data are available }\end{array}$} \\
\hline \multirow{2}{*}{\multicolumn{2}{|c|}{ Sub-Saharan Africans }} & \multicolumn{2}{|c|}{ Use European data until } \\
\hline & & \multicolumn{2}{|c|}{ more specific data are available } \\
\hline \multicolumn{2}{|c|}{ Eastern Mediterranean and Middle East } & $\begin{array}{l}\text { Use European data until mo } \\
\text { pc }\end{array}$ & $\begin{array}{l}\text { ic data are available (Arab) } \\
\text { s }\end{array}$ \\
\hline \multicolumn{4}{|c|}{ 5. Harmonized Definition (2009) [3] } \\
\hline \multirow{2}{*}{\multicolumn{2}{|c|}{ Organization (Reference) }} & \multicolumn{2}{|c|}{$\begin{array}{c}\text { Recommended Waist Circumference Threshold for Abdominal } \\
\text { Obesity }\end{array}$} \\
\hline & & Men & Women \\
\hline Europid & IDF & $\geq 94 \mathrm{~cm}$ & $\geq 80 \mathrm{~cm}$ \\
\hline \multirow[t]{2}{*}{ Caucasian } & WHO & $\geq 94 \mathrm{~cm}$ (increased risk) & $\geq 80 \mathrm{~cm}$ (increased risk) \\
\hline & & $\geq 102 \mathrm{~cm}$ (still higher risk) & $\geq 88 \mathrm{~cm}$ (still higher risk) \\
\hline United States & AHA/NHLBI (ATP III) & $\geq 102 \mathrm{~cm}$ & $\geq 88 \mathrm{~cm}$ \\
\hline Canada & Health Canada & $\geq 102 \mathrm{~cm}$ & $\geq 88 \mathrm{~cm}$ \\
\hline European & European Cardiovascular Societies & $\geq 102 \mathrm{~cm}$ & $\geq 88 \mathrm{~cm}$ \\
\hline Asian (including Japanese) & IDF & $\geq 90 \mathrm{~cm}$ & $\geq 80 \mathrm{~cm}$ \\
\hline Asian & WHO & $\geq 90 \mathrm{~cm}$ & $\geq 80 \mathrm{~cm}$ \\
\hline Japanese & Japanese Obesity Society & $\geq 85 \mathrm{~cm}$ & $\geq 90 \mathrm{~cm}$ \\
\hline China & Cooperative Task Force & $\geq 85 \mathrm{~cm}$ & $\geq 80 \mathrm{~cm}$ \\
\hline Middle East, Mediterranean & IDF & $\geq 94 \mathrm{~cm}$ & $\geq 80 \mathrm{~cm}$ \\
\hline Sub-Saharan African & IDF & $\geq 94 \mathrm{~cm}$ & $\geq 80 \mathrm{~cm}$ \\
\hline Ethnic Central and South American & IDF & $\geq 90 \mathrm{~cm}$ & $\geq 80 \mathrm{~cm}$ \\
\hline
\end{tabular}



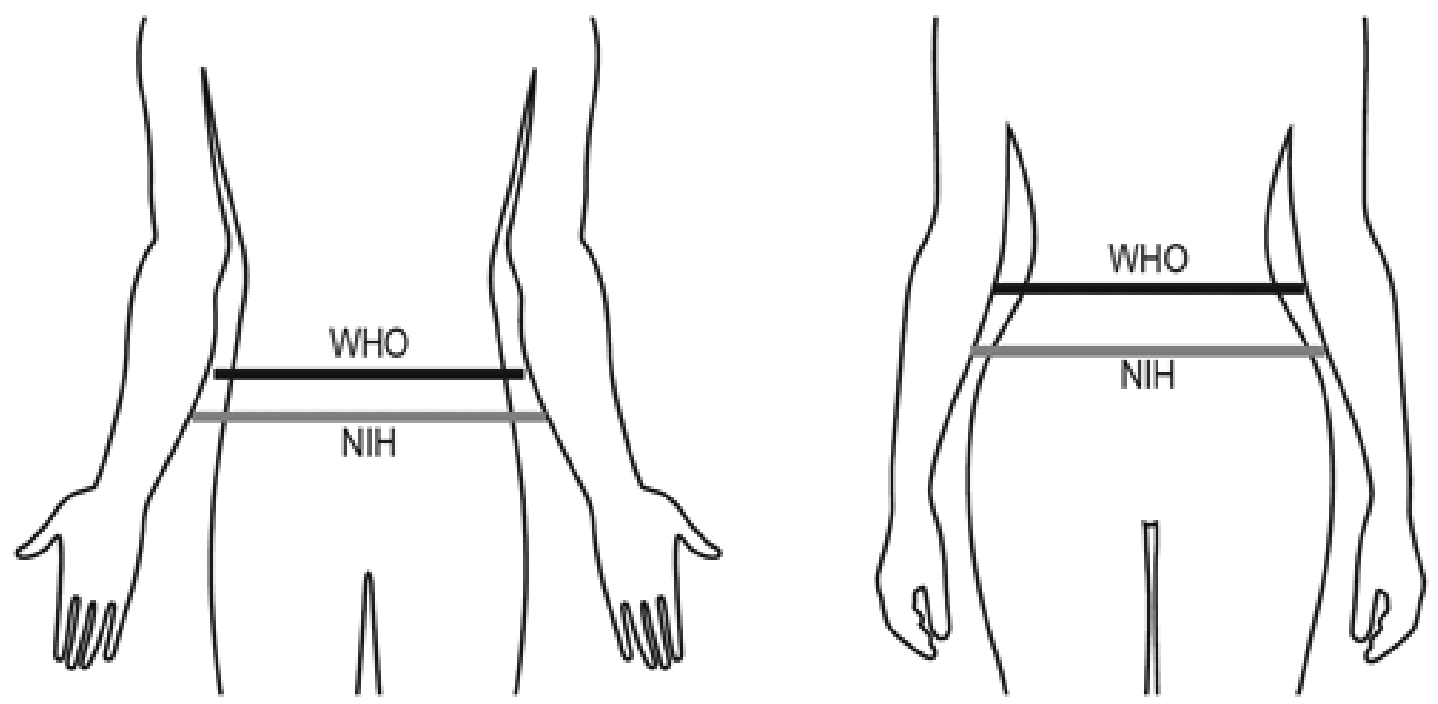

Figure 1: Waist circumference measurement sites for men and women based on World Health Organization (WHO) and National Institutes of Health (NIH) protocols ${ }^{14}$

Note: Following the WHO protocol, measure is taken midway between the highest point of the iliac crest and the bottom of the ribcage. Following the $\mathrm{NIH}$ protocol, the measure is taken at the highest point of the iliac crest.

out the best WC site out of 10 site, corresponding to the abdominal fat measured by DEXA scan, they concluded that best surrogate measure of abdominal fat was waist circumference measured $2.5 \mathrm{~cm}$ above the umbilicus. $^{12} \mathrm{WHO}^{4}$ \& IDF guidelines ${ }^{7}$ recommend measurement of waist circumference at mid- point between the lowest coastal margin \& superior border of iliac crest while NIH, NHLBI ${ }^{4}$ and NCEP/ATPIII guidelines, ${ }^{11}$ recommend measurement at the upper-most point of the iliac crest and as shown in figure 1. Based on the WC measurement locations recommended by IDF \& NCEP-ATPIII, Ma et al. conducted a prospective study on 1898 Asian subjects to compare the performance of the two locations. ${ }^{5}$ The study found that IDF location i.e. mid-point between lowest rib \& the iliac crest was better measure of central obesity and correlated better with hypertension, T2DM, metabolic syndrome \& VFA (visceral fat area) than the NCEP-ATPIII location i.e. at iliac crest. The study also found that the variation in correlation at the two locations was more marked in females as compared to males. ${ }^{5}$

\section{Problem in Indian Perspective}

For a country like India where more than $2 / 3^{\text {rd }}$ of the total population still lives in villages and nearly $1 / 4^{\text {th }}$ of the population is still illiterate determining the right site and correct measurement of waist circumference is a real challenge. In our Indian society, religious beliefs and cultural traditions like 'Purdah' and not allowing the measurement of one's body are still deeply enrooted which pose practical difficulties in waist measurement particularly in case of females. This all leads to imprecise waist circumference measurements \& creates a gender bias in accuracy and reporting. Some Indian studies tried to find out the correct cut-off for waist circumference measurement but unfortunately came up with different results. In 2005, A. Misra et al. conducted a large epidemiological study involving 883 male and 1167 female subjects. ${ }^{15}$ The group measured Body mass index (BMI), WC, waist-to-hip circumference ratio, blood pressure, and fasting samples for bloodglucose, total cholesterol, serum triglycerides, and high-density lipoprotein cholesterol in the cohort population. The study group concluded that in males, a WC cutoff point of $78 \mathrm{~cm}$ (sensitivity $74.3 \%$, specificity $68.0 \%$ ), and in females, a cutoff point of $72 \mathrm{~cm}$ (sensitivity 68.7\%, specificity $71.8 \%$ ) were appropriate in identifying those with at least one cardiovascular risk factor and for identifying those with a BMI $>21 \mathrm{~kg} / \mathrm{m}^{2}$. They further concluded that WC levels of $\geq 90$ and $\geq 80 \mathrm{~cm}$ for men and women, respectively, identified high odds ratio for cardiovascular risk factor(s) and BMI level of $>25 \mathrm{~kg} / \mathrm{m}^{2}$. In a recent study by Pratyush DD (2012) the same issue of waist circumference cut off in Asian Indian population was studied on 349 males and 364 females. ${ }^{16}$ They came to a conclusion that WC cut-off points for males was $90 \mathrm{~cm}$ with a sensitivity andspecificity of $71 \%$ and $96 \%$, respectively, and for females was $85 \mathrm{~cm}$ with a sensitivity and specificity of $86 \%$ and $93 \%$, respectively, associated with the risk factors of Metabolic Syndrome.

\section{CONCLUSION}

Waist circumference is an inexpensive \& simple tool for assessment of abdominal obesity. Since different ethnicities have different genetic makeup along with different body fat content and distribution so single universal cut off for waist circumference can't be used. Site of waist measurement is also as important as the magnitude especially in case of females. Waist circumference cut offs should be according to the ethnicities and it should be measured just above the iliac crest based on the latest harmonizing definition of the metabolic syndrome till further research. 


\section{ACKNOWLEDGEMENT}

We owe thanks to the patient and her relatives for having patience and their contribution to this undertaking.

\section{CONFLICT OF INTEREST}

None.

\section{REFERENCES}

1. Kassi E, Pervanidou P, Kaltsas G, Chrousos G. Metabolic syndrome: definitions and controversies. BMC Med. 2011;9(1):48.

2. Balkau B, Charles MA. Comment on the provisional report from the WHO consultation. European Group for the Study of Insulin Resistance (EGIR) Diabet Med. 1999;16(5):442-3.

3. Alberti KG, EckelRH, Grundy SM, ZimmetPZ, CleemanJI, DonatoKA, Fruchart JC, James WP, Loria CM, Smith SC Jr. Harmonizing the metabolic syndrome: a joint interim statement of the International Diabetes Federation Task Force on Epidemiology and Prevention; National Heart, Lung and Blood Institute; American Heart Association; World Heart Federation; International Atherosclerosis Society; and International Association for the Study of Obesity. Circulation. 2009;120(16):1640-5.

4. Cornier MA, Després JP, Davis N, Grossniklaus DA, Klein S, Lamarche B et al Assessing adiposity: a scientific statement from the American Heart Association. Circulation. 2011;124(18):1996-2019.

5. Chuang LM, Lin JW, Wei JN, Li HY. Measurement of Waist Circumference Mid-abdominal or lliac Crest? Diabetes care. 2013;36(6):1660-6.

6. Peter T. Katzmarzyk, lan Janssen, Robert Ross, Timothy S. Church, Steven N. Blair. The Importance of Waist Circumference in the Definition of Metabolic Syndrome: Prospective analyses of mortality in men, Diabetes Care. 2006;29(2):404-9.

7. Alberti KG, Zimmet P, Shaw J. Metabolic syndrome-a new world-wide definition. A Consensus Statement from the International Diabetes Federation. Diabet Med 2006;23(5):469-80.

8. Wang J, Thornton JC, Bari S, Williamson B, Gallagher D, Heymsfield SB, Horlick $M$, Kotler D, Laferrère B, Mayer L, Pi-Sunyer FX. Comparisons of waist circumferences measured at 4 sites. The American journal of clinical nutrition. 2003;77(2):379-84

9. Balkau B, Charles MA, for the European Group for the Study of Insulin Resistance (EGIR) Comment on the provisional report from the WHO consultation. Diabetic Medicine. 1999;16(5):442-3.

10. Expert Panel on Detection, Evaluation. Executive summary of the third report of the National Cholesterol Education Program (NCEP) expert panel on Detection, Evaluation, and Treatment of high blood cholesterol in adults (Adult Treatment Panel III). JAMA. 2001;285(19):2486.

11. Grundy SM, Cleeman JI, Daniels SR, Donato KA, Eckel RH, Franklin BA et al. Diagnosis and management of the metabolic syndrome: an American Heart Association/National Heart, Lung, and Blood Institute scientific statement. Circulation. 2005;112(17):2735-52

12. Guerra RS, AmaralTF, Marques EA, Mota J, Restivo MT: Anatomical location for waist circumference measurement in older adults: a preliminary study. Nutricionhospitalaria: organooficial de la Sociedad Espanola de Nutricion Parenteral y Enteral. 2012;27(5):1554-61.

13. Mason C, Katzmarzyk PT. "Waist circumference thresholds for the prediction of cardiometabolic risk: is measurement site important\&quest."European journal of clinical nutrition. 2010;64(8):862-7.

14. WHO (2011) Waist Circumference and Waist-Hip Ratio: Report of a WHO Expert Consultation, Geneva, 8-11 December 2008. Technical report, World Health Organization.

15. Misra A, Vikram NK, Gupta R, Pandey RM, Wasir JS, Gupta VP. Waist circumference cutoff points and action levels for Asian Indians for identification of abdominal obesity. International journal of obesity. 2006;30(1):106-11.

16. Pratyush DD, Tiwari S, Singh S, Singh SK. Waist circumference cutoff and its importance for diagnosis of metabolic syndrome in Asian Indians: a preliminary study. Indian journal of endocrinology and metabolism. 2012;16(1):112

Cite this article : Syed Mohd Razi Gutch Manish, Keshav GK, Sukriti K, Gupta A. Site or Size of Waist Circumference, Which one is More important in Metabolic Syndrome?. Int. J. Med. Public Health, 2016; 6(2):69-72. 